\title{
The impact of age and clinical factors on quality of life in early breast cancer: An analysis of 2208 women recruited to the UK START Trial (Standardisation of Breast Radiotherapy Trial)
}

\author{
Penelope Hopwood ${ }^{a, *}$, Joanne Haviland ${ }^{b}$, Judith Mills ${ }^{b}$, Georges Sumo ${ }^{b}$, \\ Judith M Bliss ${ }^{\mathrm{b}}$
}

${ }^{a}$ Christie Hospital NHS Trust, Wilmslow road, Withington, Manchester M20 4BX, UK

${ }^{\mathrm{b}}$ Institute of Cancer Research, Sutton, Surrey, SM2 5NG, UK

Received 22 June 2006; received in revised form 9 November 2006; accepted 20 November 2006

\section{KEYWORDS \\ Early breast cancer; Quality of life; Body image; Sexual functioning; Chemotherapy; Radiotherapy}

\begin{abstract}
Summary Quality of life (QOL) assessments of women entering a UK randomised trial of adjuvant radiotherapy (START) were investigated to estimate the independent effects on QOL of age, time since surgery, type of breast surgery, chemotherapy and endocrine therapy. QOL was evaluated using the EORTC general cancer QOL scale (EORTC QLQ-C30), breast cancer module (BR23), the Body Image Scale (BIS) and the Hospital Anxiety and Depression Scale (HADS). Independent effects of age and clinical factors were tested using multiple regression analysis.

A total of 2208 (mean age 56.9 years, range 26-87) consented to the QOL study prior to radiotherapy; $17.1 \%$ had undergone mastectomy $(M x)$ and the remainder had undergone a wide local excision (WLE). 33.3\% had received adjuvant chemotherapy (CT) and $56.7 \%$ were taking endocrine therapy (ET). Age had significant effects on QOL with older and younger subgroups predicting poorer QOL for different domains. CT affected most QOL domains and resulted in worse body image, sexual functioning, breast and arm symptoms $(<0.001)$. Mx was associated with greater body image concerns $(p<0.001)$, and WLE with more arm symptoms $(p=0.01)$. There were no effects of ET on QOL. Women $<50$ years (proxy pre-menopausal) had worse QOL in respect of anxiety, body image and breast symptoms but age and clinical factors had no effect on depression.

Overall, QOL and mental health were favourable for most women about to start RT, but younger age and receiving CTwere significant risk factors for poorer QOL, and so patients in these subgroups warrant further monitoring. Surgery had a limited impact and ET had no effect on QOL.

(c) 2006 Elsevier Ltd. All rights reserved.
\end{abstract}

*Corresponding author. Tel.: +44161446 8004; fax: +44 1614468565 .

E-mail address: penny.hopwood@christie-tr.nwest.nhs.uk (P. Hopwood). 


\section{Introduction}

Women with primary breast cancer usually undergo breast surgery plus adjuvant systemic treatments in order to reduce the risks of local and distant disease recurrence, but with the potential for multiple effects on quality of life (QOL). The overall psychosocial impact of these modern treatments has been reported. ${ }^{1-8}$ Younger patients are more likely to suffer adverse effects because of induction of an early menopause and possible infertility. Other reported problems have included poor sexual functioning, altered body image, fatigue and difficulty with shoulder and arm movements. These effects may have an impact on $\mathrm{QOL}$ in the longer term. Radiotherapy is known to have late effects on normal tissues which could contribute adversely to body image and related $\mathrm{QOL}$ parameters but it is often impossible to attribute $\mathrm{QOL}$ effects, such as breast symptoms, to a specific adjuvant strategy in multimodal therapy. ${ }^{9-15}$ Ganz and colleagues commented that women are generally well prepared for the acute toxicities of breast cancer treatments but that clinicians have limited information on the physical and psychosocial sequelae of primary treatments, or the pattern of recovery. This highlights the need for more precise data on individual treatment effects and interactions. ${ }^{16}$

The QOL protocol in the Standardisation of Breast Radiotherapy Trial (START) trial will help address these deficiencies, through its prospective design and long term follow-up, by using patient assessed measures of body image and QOL, and by identifying the effects of individual treatments in multimodal therapy. The trial includes women of all ages allowing the effect of age on QOL to be explored. The purpose of the trial is to test the effects of radiotherapy fractions $>2.0 \mathrm{~Gy}$ on local tumour control and late normal tissue response in the breast area after tumour excision. The trial comprises two randomised comparisons, namely Trial A, which tests three fractionation schedules; $50 \mathrm{~Gy}$ in 25 fractions (F) over 5 weeks versus two dose levels of a test schedule giving $41.6 \mathrm{~Gy}$ or $39 \mathrm{~Gy}$ in $13 \mathrm{~F}$ over 5 weeks, and Trial B, which tests the standard therapy (50 Gy in $25 \mathrm{~F}$ ) against a dose level of $40 \mathrm{~Gy}$ in $15 \mathrm{~F}$ over 3 weeks. ${ }^{17}$ Long-term follow-up is now being completed and QOL will form part of the outcome assessment of the randomised comparison. Over 2000 women (>50\% of trial participants) have been accrued to the QOL study, making this the largest such investigation in the UK. Prior to breaking the randomisation code, we have undertaken this analysis to describe $\mathrm{QOL}$ in the overall cohort of patients and to investigate the effects on QOL of age and other primary treatments, prior to starting radiotherapy, in order to gauge the extent to which key domains such as body image may be affected by other primary treatments. The size of the database allows us to explore the individual impact of type of surgery, endocrine therapy (ET), chemotherapy (CT), as well as examining age effects, whilst controlling for time since surgery, on all QOL domains.

\section{Aims of baseline analysis}

To describe all aspects of QOL at baseline, i.e. prior to undergoing radiation therapy.

To determine the impact on QOL of age, type of breast surgery (Mx vs WLE), adjuvant CT and ET whilst controlling for time elapsed since surgery. These factors were selected to accurately determine the impact of primary breast cancer treatment on QOL before radiotherapy, whilst taking account of the fact that patients undergoing CT would have a longer time between breast surgery and radiotherapy compared with those proceeding directly to radiotherapy. Age was included because it is known to influence some aspects of QOL.

To compare women under 50 years with those aged $\geqslant 50$ in terms of QOL. These age-groups were chosen to provide subgroups representing proxy pre- and post-menopausal status in the analysis at baseline.

\section{Methods}

Women prescribed postoperative radiotherapy for early breast cancer attending the 35 centres participating in the START trial were eligible for the QOL study. A total of 4451 patients were recruited into the START trial between 1999 and 2002. The protocol did not impose a limit on the time between surgery and randomisation into the trial, but there had to be a minimum of two weeks between the end of chemotherapy and the start of radiotherapy treatment. Patients from 31 of the centres were recruited into the QOL study, after obtaining written informed consent. To avoid introducing bias from knowledge of randomised treatment allocation, baseline $\mathrm{QOL}$ data were collected prior to randomisation into the START trial, together with demographic data.

\section{QOL Measures}

Standardized self-report QOL measures were used, including the EORTC QLQ-C $30,{ }^{18}$ the EORTC BR23, ${ }^{19}$ the Body Image Scale (BIS), ${ }^{20}$ and the Hospital Anxiety and Depression Scale (HADS). ${ }^{21}$ These 
measures were selected as they had been used successfully in other national and European breast cancer trials, allowing comparison of results.

The QLQ-C3O is a 30 -item multi dimensional cancer specific QOL scale made up of 5 functional subscales (physical, role, emotional, cognitive, and social functioning), together with 9 symptom subscales/items (fatigue, nausea and vomiting, pain, dyspnoea, insomnia, appetite loss, constipation, diarrhoea, and financial difficulties). Two further items assess global health and global quality of life.

The BR23 breast cancer module is designed to capture effects due to treatment. Its 23 items are organised into four symptom scales (systemic therapy side effects, breast symptoms, arm symptoms, and upset by hair loss) and four functional subscales (body image, sexual functioning, sexual enjoyment, and future perspective).

The BIS is a 10 -item scale designed for use with cancer patients. Items cover change in physical and sexual attractiveness, physical appearance when dressed and naked, satisfaction with the body and scars, body integrity and avoidance of people. The BIS is widely used in national breast cancer trials and psychosocial studies. The four body image items in BR23 were taken from the 10-item scale but there is no duplication of body image items in the QOL assessment booklet.

The HADS is a 14-item scale designed for use in a medical setting. It is the most widely used measure of psychological distress in cancer patients and has been used extensively in UK cancer therapy trials over many years. It has the advantage over QOL subscales that measure emotional functioning in providing an estimate of the clinical level of distress by applying threshold scores for normal, borderline or probable case levels of anxiety or depression.

Additional protocol specific symptom items were included to cover expected effects of radiotherapy and will be reported later.

\section{Scoring and statistical analysis}

\section{EORTC QLQ-C30 and BR-23}

All items were scored using a 4-point response scale, except for global health and global quality of life, for which a 7-point range was used. For the functional and global health/QL subscales, higher scores indicate a better level of functioning, whereas for the symptom scales, higher scores represent more symptoms.

Summary scores were calculated according to the EORTC manual. ${ }^{22}$ Summary statistics were calculated for baseline scores, according to categories of type of surgery (Mx, WLE), time since surgery, CT, ET and age. Subscale scores were compared between groups of patients defined according to age and the set of clinical variables tested using $t$ tests and analysis of variance (ANOVA) as appropriate. In order to investigate the effect of confounding on observed associations with QOL scores, multiple regression was used, and the significance of variables tested using the $F$-test from an ANOVA that included all relevant independent variables (surgery, time since surgery, CT, ET and age). Hence the $p$-values from the $F$-test indicate the significance of each variable after allowing for the effects of the other variables listed. Variables which remain significant in the multiple regression can be said to have independent statistically significant effects on the outcome in question.

As the majority of trial participants did not require adjuvant $C T$ and because of the observed extensive effect of $\mathrm{CT}$ on $\mathrm{QOL}$, the regression analysis was repeated including pair-wise interactions between CT and the other independent variables. A further subgroup analysis investigated associations between clinical variables and $\mathrm{QOL}$ parameters separately for age $<50$ and $\geqslant 50$ years, to differentiate between factors affecting pre- and post-menopausal women, respectively. For all other analyses age was treated as a continuous variable. Missing items have been scored according to EORTC QLQ-C30 scoring manual. ${ }^{22}$

\section{Body image scale (BIS)}

The 10 BIS item scores from 0 ('none') to 3 ('very much') were summed to give an overall total between 0 (best body image) and 30 (worst body image). The same methods for describing data and inter-group comparison were used as above.

\section{Hospital anxiety and depression scale (HADS)}

The HADS anxiety and depression subscale scores were categorised as 0-7 (normal), 8-10 (borderline anxious/depressed) and $\geqslant 11$ (probable case anxiety/depression) according to recommended thresholds. ${ }^{21,23}$ Comparisons between categories of the prognostic factors listed above were made using the appropriate $\chi^{2}$-test. In order to investigate the effect of confounding on observed associations, multiple logistic regression was used. The model included all prognostic factors listed above, and the outcome variables of anxiety and depression were grouped into two categories for the multiple logistic regression analysis $(0-7$ vs. $\geqslant 8)$. The numbers of patients scoring $\geqslant 11$ were small, which precluded using all three categories of anxiety and depression in a multiple regression. The $p$-values from the multiple logistic regression indicate the significance of each variable after allowing for the effects of the other variables listed. 
The distributions of many of the QOL subscales were skewed, but no satisfactory transformation could be found to normalise the data. Means and standard deviations (SD), together with the median and interquartile range (IQR) have been presented in order to facilitate comparison with other published data. The univariate analyses were repeated using non-parametric tests, and similar results were obtained (data not presented). To make some allowance for chance findings due to the large number of significance tests carried out, the cut-off for statistical significance was set to a more stringent level at $p=0.01$.

\section{Results}

A total of 2180 patients ( $98.7 \%$ of women accrued) provided baseline data. Reasons for non-compliance included change of treatment decision, withdrawal from trial and failure to complete questionnaires. Completeness of responses on $\mathrm{QOL}$ scales was $99 \%$, apart from the BIS, which was $96 \%$.

The geographic and age distributions of START QOL patients were comparable with the national data for breast cancer in the UK per year; ${ }^{24}$ details of ethnic groups were not available as this information was not routinely collected in the trial. Patient and clinical characteristics at baseline are shown in Table 1. Three quarters of the patients were randomised within 19.6 weeks of surgery; of

Table 1 Demographic and clinical characteristics of 2208 patients in the START QOL study.

\begin{tabular}{ll}
\hline Patient characteristic & Number of patients (\%) \\
\hline Age & $11(0.5)$ \\
$20-29$ & $116(5.3)$ \\
$30-39$ & $391(17.7)$ \\
$40-49$ & $858(38.9)$ \\
$50-59$ & $573(26.0)$ \\
$60-69$ & $241(10.9)$ \\
$70-79$ & $18(0.8)$ \\
$80-89$ & $56.9(10.4)$ \\
Mean (SD) & $56.5(50.5-63.9)$ [26-87] \\
Median (IQR) [range] & \\
& \\
Surgery & $1831(82.9)$ \\
Wide local excision* & $377(17.1)$ \\
Mastectomy & \\
Time since surgery (weeks) \\
0-4 & $450(20.4)$ \\
5-9 & $799(36.2)$ \\
10-19 & $417(18.9)$ \\
$>$ 20 & $542(24.5)$ \\
Median (IQR) & $8.9(5.6-19.6)$ \\
&
\end{tabular}

Table 1 (continued)

\begin{tabular}{|c|c|}
\hline Patient characteristic & Number of patients (\%) \\
\hline \multicolumn{2}{|l|}{ Side of primary } \\
\hline Left & 1147 (51.9) \\
\hline Right & $1061(48.1)$ \\
\hline \multicolumn{2}{|c|}{ Pathological tumour size $(\mathrm{cm})$} \\
\hline $0-0.9$ & $296(13.4)$ \\
\hline $1.0-1.9$ & $1002(45.4)$ \\
\hline $2.0-2.9$ & $610(27.6)$ \\
\hline$>3.0$ & $283(12.8)$ \\
\hline Unknown & $17(0.8)$ \\
\hline \multicolumn{2}{|l|}{ Tumour grade } \\
\hline 1 & $516(23.4)$ \\
\hline 2 & $1030(46.6)$ \\
\hline 3 & $611(27.7)$ \\
\hline Unknown & $51(2.3)$ \\
\hline \multicolumn{2}{|l|}{ Histological type } \\
\hline Ductal & $1722(78.0)$ \\
\hline Lobular & $282(12.8)$ \\
\hline $\begin{array}{l}\text { Mixed ductal and } \\
\text { lobular }\end{array}$ & $47(2.1)$ \\
\hline Special types ${ }^{\dagger}$ & $113(5.1)$ \\
\hline Not reported & $44(1.9)$ \\
\hline \multicolumn{2}{|l|}{ Node status } \\
\hline Positive & $692(31.3)$ \\
\hline Negative & $1444(65.4)$ \\
\hline Unknown & $72(3.3)$ \\
\hline \multicolumn{2}{|l|}{ Axillary surgery } \\
\hline None & $64(2.9)$ \\
\hline Axillary clearance & $1518(68.8)$ \\
\hline Axillary sampling & $584(26.4)$ \\
\hline Sentinel node biopsy & $38(1.7)$ \\
\hline Unknown & $4(0.2)$ \\
\hline \multicolumn{2}{|l|}{ Adjuvant treatment } \\
\hline None & $177(8.0)$ \\
\hline Endocrine therapy & $1296(58.7)$ \\
\hline Chemotherapy & $221(10.0)$ \\
\hline $\begin{array}{l}\text { Endocrine therapy } \\
\text { and chemotherapy }\end{array}$ & $514(23.3)$ \\
\hline
\end{tabular}

*WLE includes patients who had undergone quadrantectomy (1), partial mastectomy (4), lumpectomy (1) and radiologically guided excision biopsy (8).

${ }^{\dagger}$ Special types include tubular and medullary tumours.

$\$ 40$ patients received endocrine therapy with drugs other than tamoxifen.

${ }_{2} 2$ patients received endocrine therapy with drugs other than tamoxifen.

the $542(25 \%)$ randomised more than 20 weeks following surgery, almost all received adjuvant chemotherapy (CT) prior to radiotherapy. The protocol required a minimum of 2 weeks between the end of CT and start of radiotherapy: the median interval (IQR) was 35 (25-56) days. 
Three significant interactions were observed between type of treatment and time from surgery. First, patients not receiving chemotherapy were randomised earlier than those receiving it (median 6.7 weeks versus 24.1 weeks, $p<0.001$ ). Second, patients undergoing wide local excision (WLE) were randomised earlier than those having mastectomy (median 8.3 weeks versus 16.8 weeks, $p<0.001$ ). Third, mastectomy patients were more likely to receive chemotherapy than those who had wide local excision $(62.7 \%$ versus $28.5 \%, p<0.001)$.

Key QL domains: body image, sexual functioning and psychological distress

\section{Body image (BIS)}

Over $50 \%$ women had no body image concerns (summary scores of zero) as shown in Fig. 1. However, scores for women undergoing mastectomy (Mx) (median 7; (IQR 3-12)) were significantly worse than those treated by WLE (median 3; (IQR $0-7), p<0.001)$. Further, scores for women on CT, (with or without concomitant ET), were significantly higher than those women not receiving systemic therapy (ST), (CT: median 7.0; (IQR 3-13); vs. no ST: median 2.0; (IQR 0-6), $p<0.001$ ). Favourable BIS scores were also seen for women who received endocrine therapy alone (median 2.0; (IQRO-5)).

The impact of CT was largely explained by the effect of distress due to hair loss; when CT and the item 'distress over hair loss' were both included in a regression model for BIS, only the effect for 'distress due to hair loss' remained significant. In multiple regression, type of surgery, age and CT remained highly statistically significant (all $p<0.001$ ), as shown in Table 2 .

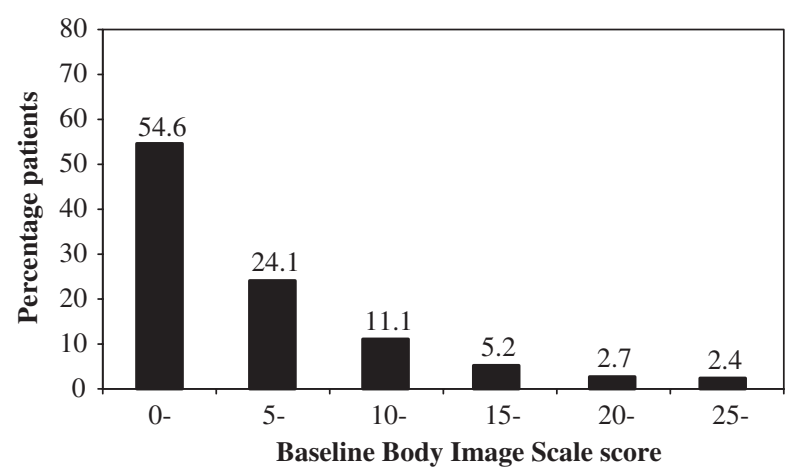

Figure 1 Distribution of baseline Body Image Scale summary scores (higher scores indicate worse body image).
Sexual functioning (SEF) and sexual enjoyment (SEE), (BR23)

Of 2087 patients who completed the sexual functioning questions $(95.7 \%$ of all patients completing BR23), 1164 (56\%) reported having no interest in sex and 54\% reported being sexually inactive. Descriptive statistics are shown in Table 3. Of 848 sexually active patients, $5 \%$ reported that sex was not enjoyable, whereas enjoyment was described as 'a little', 'quite a bit' or 'very much' by $34 \%, 39 \%$ and $22 \%$ women, respectively.

Allowing for all variables in the multiple regression, age and having chemotherapy were significant $(p<0.001$ for SEF and $p=0.006$ for SEE). Of note, there was no independent effect of type surgery on sexual functioning (Table 2 ).

\section{Anxiety and depression (HADS)}

The levels of anxiety and depression were calculated for each subscale. The median anxiety subscale score was 5.0 (IQR 3-8) and the median depression score was 2 (IQR 1-5). The prevalence of anxiety was higher than for depression; two thirds $(1468,67.6 \%)$ of patients scored in the normal range for anxiety, 391 (18.0\%) were in the borderline range and $313(14.4 \%)$ were probable cases, whereas $88.1 \%$ of patients $(n=1910)$ scored in the normal range for depression, 8.9\% (192) were in the borderline range and $3.1 \%$ (67) were probable cases. There was a higher percentage of 'cases' in patients who had received CT $(p<0.001)$. In multiple regression, low anxiety was associated with increased age but no factors had a significant effect on depression scores (Table 2).

\section{Physical domains: breast and arm symptoms (EORTC BR23), physical functioning (EORTC QLQ-C30)}

\section{Breast symptoms}

Almost two thirds of patients reported pain in the breast area, 34.9\% had swelling in the breast area, over half reported over-sensitivity and $22 \%$ had skin problems (Table 3). In the multiple regression analysis, time since surgery, age and CT were significantly associated with breast symptoms as shown in Table 2. Type of surgery and ET had no effect.

\section{Arm symptoms}

Significant effects were observed for type of surgery (fewer symptoms for $M x$ patients, $p=0.01$ ), time since surgery (fewer symptoms with increased time since surgery, $p<0.001$ for 
Table 2 Impact of clinical factors and age on baseline QOL parameters.

\begin{tabular}{|c|c|c|c|c|c|}
\hline $\begin{array}{l}\text { Functional subscale or } \\
\text { symptoms item }\end{array}$ & $\begin{array}{l}\text { Type of } \\
\text { surgery }\end{array}$ & $\begin{array}{l}\text { Time since } \\
\text { surgery }\end{array}$ & Chemotherapy & $\begin{array}{l}\text { Endocrine } \\
\text { therapy }\end{array}$ & Age \\
\hline \multicolumn{6}{|c|}{ EORTC QLQ-C30 functional subscale: } \\
\hline Physical & 0.02 & 0.27 & 0.14 & 0.08 & $<0.001$ \\
\hline Role & 0.03 & $<0.001$ & $<0.001$ & 0.63 & 0.38 \\
\hline Emotional & 0.58 & 0.28 & 0.23 & 0.78 & $<0.001$ \\
\hline Cognitive & 0.94 & 0.06 & 0.29 & 0.86 & 0.002 \\
\hline Social & 0.65 & 0.006 & $<0.001$ & 0.36 & $<0.001$ \\
\hline \multicolumn{6}{|l|}{ EORTC QLQ-C30 symptoms: } \\
\hline Fatigue & 0.56 & 0.16 & $<0.001$ & 0.17 & 0.66 \\
\hline Nausea/vomiting & 0.24 & 0.78 & $<0.001$ & 0.25 & 0.01 \\
\hline Pain & 0.58 & $<0.001$ & 0.01 & 0.26 & 0.005 \\
\hline Dyspnoea & 0.72 & 0.01 & $<0.001$ & 0.45 & $<0.001$ \\
\hline Insomnia & 0.72 & 0.59 & 0.95 & 0.17 & $<0.001$ \\
\hline Appetite loss & 0.01 & 0.80 & 0.01 & 0.51 & 0.99 \\
\hline Constipation & 0.46 & 0.10 & $<0.001$ & 0.81 & 0.04 \\
\hline Diarrhoea & 0.04 & 0.006 & 0.002 & 0.20 & 0.54 \\
\hline Financial difficulties & 0.90 & 0.68 & 0.07 & 0.67 & $<0.001$ \\
\hline Global health & 0.96 & 0.14 & $<0.001$ & 0.11 & 0.73 \\
\hline Global QL & 0.30 & 0.02 & $<0.001$ & 0.12 & 0.54 \\
\hline \multicolumn{6}{|l|}{ EORTC BR-23: } \\
\hline Sexual functioning & 0.03 & 0.14 & $<0.001$ & 0.23 & $<0.001$ \\
\hline Sexual enjoyment & 0.96 & 0.77 & 0.006 & 0.96 & $<0.001$ \\
\hline $\begin{array}{l}\text { Systemic therapy side } \\
\text { effects }\end{array}$ & 0.19 & 0.36 & $<0.001$ & 0.54 & 0.08 \\
\hline Upset by hair loss & 0.77 & 0.001 & $<0.001$ & 0.77 & 0.03 \\
\hline Breast symptoms & 0.30 & $<0.001$ & 0.003 & 0.96 & 0.001 \\
\hline Arm symptoms & 0.007 & $<0.001$ & 0.55 & 0.05 & $<0.001$ \\
\hline Future perspective & 0.19 & 0.02 & 0.001 & 0.43 & $<0.001$ \\
\hline Body image scale (10 item) & $<0.001$ & 0.29 & $<0.001$ & 0.53 & $<0.001$ \\
\hline HADS anxiety & 0.45 & 0.26 & 0.97 & 0.48 & $<0.001$ \\
\hline HADS depression & 0.73 & 0.22 & 0.19 & 0.49 & 0.21 \\
\hline
\end{tabular}

$p$-values represent significance of $F$-test (likelihood ratio test for HADS anxiety and depression) obtained from a multiple regression model including terms for age, type of surgery, time since surgery, chemotherapy and tamoxifen.

For time since surgery and age the $p$-values correspond to trend tests.

$p$-values considered to be statistically significant $(p \leqslant 0.01)$ are highlighted in bold.

Table shows $p$-values obtained from multiple regression analysis including all treatment variables and age.

Directions of statistically significant effects were as follows:

Type of surgery: scores for appetite loss and body image concerns were worse for mastectomy patients; arm symptoms were worse for WLE patients.

Time since surgery: scores for role functioning, social functioning, pain, upset by hair loss, breast symptoms and arm symptoms improved as time from surgery increased; scores for diarrhoea, were worse with increasing time.

Chemotherapy: scores for breast symptoms were better in patients who had received chemotherapy; scores for all other significant QL parameters were worse.

Age: scores for physical functioning, sexual functioning, sexual enjoyment were worse with increasing age; scores for all other significant variables were better with increasing age.

trend), and age (fewer symptoms in the youngest and oldest women, $p=0.001$ ), (see Table 2). Shoulder stiffness (protocol item) was reported as 'a little', 'quite a bit' or 'very much' by $30 \%, 7 \%$ and $2 \%$ patients, respectively, $60 \%$ being symptom free.

Standardised item scores for the full breast cancer module are shown in Table 3.

\section{Physical functioning (EORTC QLQ C-30)}

The distribution of physical functioning (PF) scores is presented in Fig. 2 and descriptive statistics are presented in Table 4. These showed overall high levels of PF. Only older age had a significant association with worse PF in the multiple regression analysis (Table 2), although the difference in median PF scores across age categories was modest. 
Table 3 Baseline standardised scores for functional and symptom scales from EORTC BR23 Breast Cancer Module.

\begin{tabular}{lrll}
\hline Scale from EORTC BR23 & $N$ & Mean (SD) & Median (IQR) \\
\hline $\begin{array}{l}\text { Functional scales } \\
\text { Sexual function }\end{array}$ & 2087 & $19.2(23.0)$ & $16.7(0-33.3)$ \\
Sexual enjoyment & 848 & $59.1(28.2)$ & $66.7(33.3-66.7)$ \\
Future perspective & 2149 & $54.8(29.4)$ & $66.7(33.3-66.7)$ \\
Body image (4 items) & & $78.1(25.8)$ & $83.3(66.7-100.0)$ \\
Symptom scales/items & 2153 & & \\
Systemic therapy side effects & & $20.9(17.2)$ & $19.0(9.5-28.6)$ \\
Arm symptoms & 2174 & $21.2(19.8)$ & $22.2(11.1-33.3)$ \\
Upset by hair loss & 2172 & $50.6(36.2)$ & $33.3(33.3-100.0)$ \\
Shoulder stiffness & 547 & $16.8(23.7)$ & $0(0-33.3)$ \\
Breast symptoms & 2169 & $18.5(16.8)$ & $16.7(8.3-25.0)$ \\
BR23 Breast symptoms & 2170 & Item frequency & \\
Pain & $N$ & $1390(64.1 \%)$ & \\
Swelling & $N 70$ & $1190(55.0 \%)$ & \\
Sensitivity & 2155 & $488(22.5 \%)$ & \\
Skin problems & 2163 & & \\
\hline
\end{tabular}

$\mathrm{SD}$, standard deviation; IQR, interquartile range.

High scores on functional scales indicate high levels of functioning, whereas high scores on the symptom scales and items indicate high levels of symptoms.

*Four items from the BR23 have been combined with the 6 additional items that make up the 10-item Body Image Scale, reported separately in Table 4.

†Protocol item. 857 patients (39.5\%) reported some degree of shoulder stiffness.

${ }^{\ddagger}$ Individual items that comprise the breast symptoms summary score. Number (\%) of patients reporting some degree of each individual symptom are shown.

Global health and global QL (EORTC QLQ-C30)

The distribution of scores for global health and global QL showed that the majority of patients recorded good or high overall QOL. Interestingly, the only significant clinical factor to affect global health and global QOL was receiving chemotherapy (see Table 2).

\section{Other QOL domains and symptoms (EORTC QLQ- C30)}

Standardised scores for the other four functional scales (role, emotional, cognitive and social functioning) are shown in Table 4. Also shown are mean standardised scores for the symptom scales and items. Results of the multiple regression analyses are summarised in Table 2.

Most women had moderately good to high levels of functioning and scores were comparable with EORTC reference values. In multiple regression, time from surgery, CT and age were the main effects. Worse cognitive functioning and better emotional functioning were associated with increased age, but the absolute difference in scores across age was small and may have been of little or no clinical significance. For role and social func-

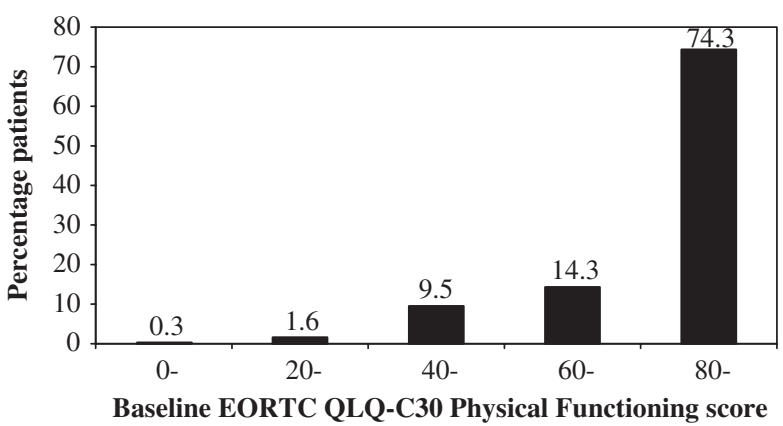

Figure 2 Distribution of baseline EORTC QLQ-C30 physical functioning subscale scores (higher scores indicate better physical functioning).

tioning, improvement was observed with increased time from surgery but worse scores were associated with CT. Increased age was associated with better social functioning but worse physical functioning, whereas $\mathrm{CT}$ rather than age was associated with increased fatigue (Table 2). Younger women reported more physical symptoms, social and financial difficulties. 
Table 4 Baseline standardised scores for functional and symptom scales from the EORTC QLQ-C30 core questionnaire.

\begin{tabular}{llrr}
\hline Scale from EORTC QLQ-C30 & $N$ & Mean (SD) & Median (IQR) \\
\hline Functional scales & & & \\
Physical functioning & 2176 & $83.8(16.7)$ & $86.7(73.3-93.3)$ \\
Role functioning & 2174 & $70.1(28.7)$ & $66.7(50.0-100.0)$ \\
Emotional functioning & 2173 & $75.3(21.6)$ & $75.0(66.7-91.7)$ \\
Cognitive functioning & 2175 & $81.9(21.0)$ & $83.3(66.7-100.0)$ \\
Social functioning & 2174 & $75.6(25.9)$ & $83.3(66.7-100.0)$ \\
Symptom scales/items & & & \\
Fatigue & 2176 & $33.1(22.7)$ & $33.3(22.2-44.4)$ \\
Nausea and vomiting & 2176 & $7.1(14.9)$ & $0(0-16.7)$ \\
Pain & 2176 & $20.9(23.9)$ & $16.7(0-33.3)$ \\
Dyspnoea & 2163 & $13.4(22.0)$ & $0(0-33.3)$ \\
Insomnia & 2164 & $33.8(31.2)$ & $33.3(0-66.7)$ \\
Appetite loss & 2171 & $12.1(23.0)$ & $0(0-33.3)$ \\
Constipation & 2164 & $14.7(24.5)$ & $0(0-33.3)$ \\
Diarrhoea & 2168 & $6.3(16.3)$ & $0(0-0)$ \\
Financial difficulties & 2171 & $15.4(27.5)$ & $0(0-33.3)$ \\
Global health & 2169 & $66.8(20.0)$ & $66.7(50.0-83.3)$ \\
Global QL & 2168 & $69.8(21.2)$ & $66.7(50.0-83.3)$ \\
\hline
\end{tabular}

SD, standard deviation; IQR, interquartile range.

High scores on functional scales indicate high levels of functioning, whereas high scores on the symptom scales and items indicate high symptom severity.

Summary of impact of age and clinical variables on QOL symptoms and domains

A summary of the effects of age and clinical variable is shown in Table 2; these effects were wide ranging involving nearly all areas of function and symptoms. Age and CT had the most extensive effects whereas ET had no significant effect on key $\mathrm{QOL}$ variables. More QOL parameters were adversely affected by younger age than older age, as detailed in the table.

\section{Subgroup analysis and interactions}

Significant interactions were observed between CT and time since surgery on the scores for physical, role and social functioning, fatigue, pain and appetite loss, breast and arm symptoms, global health and global QL. Improvements were found in fatigue, appetitive loss, global health and global QL for the NoCT group but not for the CT group. No effect of endocrine therapy on any QL domains or symptoms was found for the NoCT group.

Impact of treatment on women aged $<\mathbf{5 0}$ years Five hundred and eighteen (23.5\%) were aged under 50 years at the time of baseline assessment (broadly considered pre-menopausal). Associations between clinical variables and $\mathrm{QOL}$ parameters were examined separately for women aged $<50$ years and those aged $\geqslant 50$ years, and although no consistent patterns were found, the adverse effect of chemotherapy on sexual functioning appeared to be greater for the younger women $(p=0.04)$. However, overall, mean SEF and SEE scores were more favourable for women $<50$ (SEF: 27.2; SEE: 62.5) compared to women $\geq 50$ (SEF: 16.6, SEE: 57.3).

Psychological morbidity prevalence rates were significantly higher for younger women (anxiety: $41.5 \%$ of women $<50$ years scored $\geq 8$ compared with $29.6 \%$ of women $\geq 50$ years, $p<0.001$; depression: $14.9 \%$ of women $<50$ years scored $\geq 8$ compared with $11.0 \%$ of women $\geq 50$ years, $p=0.02$ ). BIS scores were also significantly higher for women $<50$ years (median7, (IQR 3-13)); compared with those $\geq 50$ years (median 2, (IQR $0-6), p<0.001$ ), indicating more body image concern.

\section{Discussion}

Findings from this large patient population highlight the important effects of age, type of surgery and adjuvant CT on the QOL in women about to start radiotherapy, whilst allowing for time elapsed since first treatment. Of particular note was the dominant effect of adjuvant chemotherapy on a 
wide range of QOL domains: secondary analyses showed that CT extenuated the otherwise beneficial effect of time from surgery on a range of QOL domains, so that the majority of patients who did not receive $\mathrm{CT}$ had better $\mathrm{QOL}$ over time. Interestingly, despite the deleterious effect of $\mathrm{CT}$ on QOL, there was no effect of CT on psychological distress. Patients who were younger and who had received CT also had a worse future perspective, possibly consistent with their less favourable prognosis and the wide ranging impact of treatment on them. Overall, global health/QOL ratings were only affected by $\mathrm{CT}$, emphasising the adverse impact of this systemic therapy over and above surgery or endocrine treatment for the subgroup of women who received it.

As expected, there was a clinically significant increase in body image problems for women undergoing $M x$ compared with WLE, as extensively reported in the literature. ${ }^{7,20,26,27}$ The effect of chemotherapy related hair loss on body image has been reported ${ }^{28}$ but a precise measure of its impact in comparison to other treatment effects has been lacking. It is possible that weight gain due to CT (not assessed in this trial) as well as distress due to hair loss contributed to our result. Given improvements to body image outcomes from the use of conservative surgery, it is particularly important to identify other factors that may confound this result. Mediating factors, (not assessed here), such as the importance of appearance to an individual patient, and preference for the type of breast surgery, can also determine adjustment to body image changes and should be also be taken into account clinically. Arm symptoms were significantly worse for women treated by WLE rather than $M x$, but no difference in overall physical functioning was attributable to type of surgery in the regression analysis, which is contrary to other findings. ${ }^{16}$ This may reflect the importance of controlling for other key variables when investigating these effects.

Levels of psychological distress were generally comparable with other published reports of cancer patients, ${ }^{29-32}$ and population samples, ${ }^{31,33,34}$ suggesting good psychological adaptation for the majority of women, as reported recently by Ganz et al. $^{16}$ Emotional functioning was protected by older age but unaffected by clinical factors, despite the long and complex treatments involved for some women. However, the impacts of treatment were wide ranging in other aspects of QOL.

Levels of sexual activity and enjoyment were low overall, as reported in other published data. ${ }^{7,19,25}$ Few comparison data with age-matched healthy women are available ${ }^{30}$ but results may be similar to population samples. There was no significant effect of Mx on SEF, which was unexpected given earlier studies in this area. However, details of sexual activity before breast surgery were unavailable in this trial, to exclude a change over time from diagnosis. Adjuvant CT had a significant impact on SEF and SEE, regardless of type of surgery, as reported by Ganz et al., ${ }^{16}$ particularly the sexual well-being of younger women. Younger women who remained pre-menopausal, through not having chemotherapy, had the best sexual functioning, suggesting the negative impact of a transition through an artificial menopause. These results suggest that longer-term effects reported in the literature may be apparent early in the treatment pathway.

In contrast to chemotherapy, endocrine therapy had no significant effect on QOL domains when other factors were controlled for, but this probably reflects the short prescribing time; outcomes at 2 and 5-years follow-up will be more informative.

Several QOL domains varied with time from surgery or age and these factors should be controlled for in other QOL analyses. The effect of age on anxiety and sexual activity may represent a moderating effect of age-related reporting, but $\mathrm{PF}$ and other symptoms are likely to represent more direct effects. Physical functioning was well preserved for the majority of women, with no significant worsening with chemotherapy, in contrast to other research, ${ }^{2}$ but the small age effect confirmed the findings of Fehlauer et al. ${ }^{35}$ The range of scores for other EORTC functional subscales were comparable with published reference data ${ }^{36}$ and contemporary publications. ${ }^{25}$ Frequencies of low levels of functioning were comparable to or better than reference values but the impact of co-morbidity on QOL domains may be more apparent over time and will be reported separately.

This QOL analysis is based on a large, representative sample of women in the United Kingdom and covers a wide age range, so that we can have confidence in the results. Findings represent the impact of contemporary surgical methods and adjuvant systemic treatments, in contrast to many earlier studies in this field. Care has been taken to investigate interactions to best explain the findings. A limitation in this approach is that the trial design did not afford the chance for a pre-operative assessment of QOL which may limit the interpretation of some results.

\section{Conclusions}

A broad and detailed description of QOL has been presented and the independent adverse effects of age and primary treatments on $\mathrm{QOL}$ determined, 
prior to undergoing radiotherapy. Overall $\mathrm{QOL}$ parameters were better for the majority of women who had undergone WLE and avoided CT. The impact of surgery was narrow in QOL terms compared with CT and age effects, but all of these factors can have a clinically significant impact. Treating teams need to be aware of the differential effects of age and primary treatments for breast cancer in women presenting for radiotherapy, in order to offer additional support and intervention for those at increased risk of poor QOL.

\section{Acknowledgements}

We extend grateful thanks to the clinical teams, research nurses and trial co-ordinators involved in the START trial, who recruited patients and assisted with early QOL data collection. Patients in the trial have provided high quality and quantity of data and we would like to thank all the participants. We acknowledge earlier statistical support from Caroline Harper and appreciate support and comments from the Trial Management Group: Edwin Aird, Jane Barrett, Peter Barrett-Lee, Judith Bliss, Jacqueline Brown, Jane Dobbs, John Dewar, Joanne Haviland, Penelope Hopwood, Peter Hoskin, Pat Lawton, Brian Magee, Judith Mills, David Morgan, Roger Owen, Mark Sydenham, Karen Venables, John Yarnold.

The START trial is funded by Cancer Research UK, The Medical Research Council and The Department of Health

\section{References}

1. Avis NE, Assmann SF, Kravitz HM, Ganz PA, Ory M. Quality of life in diverse groups of midlife women: assessing the influence of menopause, health status and psychosocial and demographic factors. Qual Life Res 2004;13:933-46.

2. Bloom JR, Stewart SL, Chang S, Banks PJ. Then and now: quality of life of young breast cancer survivors. PsychoOncology 2004;13:147-60.

3. Bloom JR. Surviving and thriving? Psycho-Oncology 2002;11:89-92.

4. Dorval M, Maunsell E, Deschenes L, Brisson J. Type of mastectomy and quality of life for long term breast carcinoma survivors. Cancer 1998;83:2130-8.

5. Ferrell BR, Grant M, Funk B, Otis-Green S, Garcia N. Quality of life in breast cancer Part I: Physical and social well-being. Cancer Nurs 1997;20:398-408.

6. Ganz PA, Coscarelli A, Fred C, Kahn B, Polinsky ML, Peterson L. Breast cancer survivors: psychosocial concerns and quality of life. Breast Cancer Res Treat 1996;38:183-99.

7. King MT, Kenny P, Shiell A, Hall J, Boyages J. Quality of life three months and one year after first treatment for early stage breast cancer: influence of treatment and patient characteristics. Qual Life Res 2000;9:789-800.
8. Thewes B, Butow P, Girgis A, Pendlebury S. The psychosocial needs of breast cancer survivors; a qualitative study of the shared and unique needs of younger versus older survivors. Psycho-Oncology 2004;13:177-89.

9. Amichetti M, Caffo O. Quality of life in patients with early stage breast carcinoma treated with conservation surgery and radiotherapy. An Italian monoinstitutional study. Tumori 2001;87:78-84.

10. Kenny P, King MT, Shiell A, et al. Early stage breast cancer: costs and quality of life one year after treatment by mastectomy or conservative surgery and radiation therapy. Breast 2000;9:37-44.

11. Wallace LM, Priestman SG, Dunn JA, Priestman TJ. The quality of life of early breast cancer patients treated by two different radiotherapy regimens. Clin Oncol 1993;5:228-33.

12. Wazer DE, DiPetrillo T, Schmidt-Ullrich R, et al. Factors influencing cosmetic outcome and complication risk after conservative surgery and radiotherapy for early-stage breast carcinoma. J Clin Oncol 1992;10:356-63.

13. Whelan TJ, Levine M, Julian J, Kirkbride P, Skingley P. The effects of radiation therapy on quality of life of women with breast carcinoma: results of a randomized trial. Ontario Clinical Oncology Group. Cancer 2000;88:2260-6.

14. Sneeuw KC, Aaronson NK, Yarnold JR, et al. Cosmetic and functional outcomes of breast conserving treatment for early stage breast cancer. 1. Comparison of patients' ratings, observers' ratings, and objective assessments. Radiother Oncol 1992;25:153-9.

15. Yarnold J, Ashton A, Bliss J, et al. Fractionation sensitivity and dose response of late adverse effects in the breast after radiotherapy for early breast cancer: long-term results of a randomised trial. Radiother Oncol 2005;75:9-17.

16. Ganz PA, Kwan L, Stanton AL, et al. Quality of Life at the end of primary treatment of breast cancer: first results from the moving beyond cancer randomized trial. J Natl Cancer Inst 2004;96:376-87.

17. START Trial Management Group: Standardization of Breast Radiotherapy (START) trial. Clin Oncol 1999:11:145-147 (editorial).

18. Aaronson NK, Ahmedzai S, Bergman B, et al. The European organization for research and treatment of cancer QLQ-C30: a quality-of-life instrument for use in international clinical trials in oncology. J Natl Cancer Inst 1993;85:365-76.

19. Sprangers MA, Groenvold M, Arraras JI, et al. The European organization for research and treatment of cancer breast cancer-specific quality-of-life questionnaire module: first results from a three-country field study. J Clin Oncol 1996;10:2756-68.

20. Hopwood P, Fletcher I, Lee A, Al Ghazal S. A body image scale for use with cancer patients. Eur J Cancer 2001;37: 189-97.

21. Zigmond AS, Snaith RP. The hospital anxiety and depression scale. Acta Psychiatr Scand 1983;67:361-70.

22. Fayers PM, Aaronson N, Bjordal K, et al. EORTC QLQ-C30 Scoring manual on behalf of the EORTC Quality of Life Group, 3rd Ed., 2001.

23. Hopwood P, Howell A, Maguire P. Screening for psychiatric morbidity in patients with advanced cancer of the breast: Validation of two self-report questionnaires. $\mathrm{Br} J$ Cancer 1991;64:353-6.

24. Office for National Statistics: Cancer statistics registrations 2001. HMSO 2004.

25. Rayan G, Dawson LA, Bezjak A, et al. Prospective comparison of breast pain in patients participating in a randomized trial of breast-conserving surgery and tamoxifen with or 
without radiotherapy. Int $J$ Radiat Oncol Biol Phys 2003;55:154-61.

26. Curran D, van Dongen JP, Aaronson NK, et al. Quality of life of early-stage breast cancer patients treated with radical mastectomy or breast-conserving procedures: results of EORTC Trial 10801. The European Organization for Research and Treatment of Cancer (EORTC), Breast Cancer Cooperative Group (BCCG). Eur J Cancer 1998;34:307-14.

27. Ganz PA, Schag AC, Lee JJ, et al. Breast conservation versus mastectomy: is there a difference in psychological adjustment or quality of life in the year after surgery? Cancer 1992;69:1729-38.

28. Hunt N, McHale S. The psychological impact of alopecia. $\mathrm{Br}$ Med J 2005;331:951-3.

29. Burgess C, Cornelius V, Love $S$, et al. Depression and anxiety in women with early breast cancer: five year observational cohort study. Br Med J 2005;330:702-5.

30. Helgeson V, Tomich PL. Surviving cancer: a comparison of 5year disease-free breast cancer survivors with healthy women. Psycho-Oncology 2005; 14:307-17.

31. Groenvold M, Fayers PM, Sprangers MA, et al. Anxiety and depression in breast cancer patients at low risk of recurrence compared with the general population: a valid comparison? J Clin Epidemiol 1999;52:523-30.

32. Osborne RH, Elsworth GR, Sprangers MA, Oort FJ, Hopper JL. The value of the Hospital Anxiety and Depression Scale (HADS) for comparing women with early onset breast cancer with population-based reference women. Qual Life Res 2004; 13:191-206.

33. Watson M, Haviland JS, Greer S, Davidson S, Bliss JM. Influence of psychological response on survival in breast cancer: a population-based cohort study. Lancet 1999;354:1331-6.

34. Lampic C, Thurfjell E, Bergh J, Sjoden PO. Short- and longterm anxiety and depression in women recalled after breast cancer screening. Eur J Cancer 2001;37:463-9.

35. Fehlauer F, Tribius S, Mehnert A, Rades D. Health-related quality of life in long term breast cancer survivors treated with breast conserving therapy: impact of age at therapy. Breast Cancer Res Treat 2005;92:217-22.

36. Fayers P, Weeden S, Curran D. EORTC QLQ-C30 Reference values on behalf of the EORTC Quality of Life Study Group, 1998.

Available online at www.sciencedirect.com

\section{$\because$ ScienceDirect}

